\title{
A Scheme on PN Code Tracking of DS/FH Signals for Telemetry, Tracking, and Commanding (TT\&C)
}

\author{
Shaohua Chen ${ }^{\text {a, }}$, Jiaqi $\mathrm{Li}^{\mathrm{a}}$, Ju Wang ${ }^{\mathrm{a}}$ \\ ${ }^{a}$ Department of Information and Electronics, Beijing Institute of Technology, Beijing, China
}

\begin{abstract}
The impact of Doppler frequency hopping on code tracking performance is simulated. A design on pseudonoise (PN) Code Tracking Loop aided by the carrier tracking loop according to the carrier frequency hoping pattern is proposed in this paper. This scheme can be applied in the Telemetry, Tracking, and Commanding system which uses Direct Sequence/Frequency Hopping Spread Spectrum (DS/FH) hybrid spread spectrum technology. Simulations of the code tracking loop under different carrier to noise ratios are compared based on this scheme. The results show this method on PN code tracking of DS/FH signals is valid.
\end{abstract}

Index Terms: TT\&C; Code Tracking Loop; DS/FH; Doppler Frequency Hopping

(C) 2011 Published by MECS Publisher. Selection and/or peer review under responsibility of the Research Association of Modern Education and Computer Science

\section{Introduction}

The purpose of Telemetry, Tracking and Command (TT\&C) system is ranging and velocity measurement. Ranging is realized by measuring the phase delay between the received and local pseudo-noise (PN) code. In TT\&C system Doppler frequency agility exists because of the carrier frequency hopping and this frequency agility can be compensated with some method [1]. There is also some paper analyzing the code tracking performance in the presence of residual frequency and finite front-end bandwidth. Doppler hopping brings no code Doppler shift but frequency modulation to the incoming signal, which deteriorates the cross correlation between the incoming PN code and the reference code [2].For the Direct Sequence Spread Spectrum System, some schemes aided by the carrier tracking loop on PN code tracking loop exist and they have the sufficient accuracy for ranging [3]. But this aided method is based on the fixed ratio of carrier to the code rate. The code tracking performance is analyzed in some papers [4][5] in Direct Sequence Spread Spectrum system. However, there are some influences on code tracking loop based on DS/FH hybrid spread spectrum system, because of the higher dynamics and the carrier frequency hopping. In this paper, simulations about the impact of the carrier frequency hopping on the code tracking loop performance is provided, and a scheme on the PN code tracking loop aided by the carrier under different ratios of carrier to the code rate according to frequency hopping pattern, is proposed to eliminate the impact of the frequency hopping in the Direct Sequence and Frequency Hopping hybrid spread spectrum system. Finally it is demonstrated that this method is valid in

* Corresponding author:

E-mail address: hua123@bit.edu.cn 
different carrier to noise ratios for the Telemetry, Tracking and Command (TT\&C) system based on the DS/FH hybrid spread spectrum technology.

\section{Code Tracking Loop and Signal}

\subsection{Code tracking loop}

The code tracking loop structure[3] is shown in Fig. 1. Dot product power discriminator and second-order filter are used in the delay locked loop (DLL).

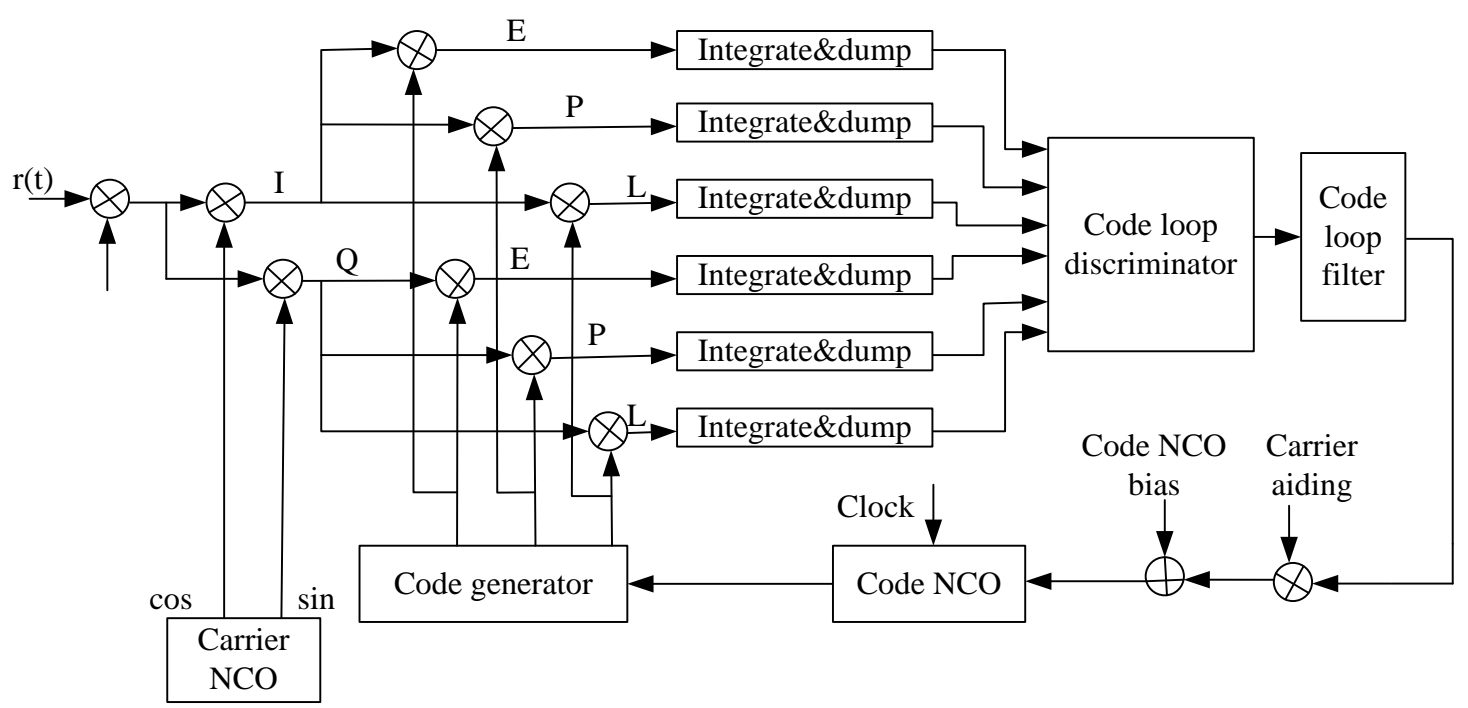

Fig. 1. Code tracking loop structure

The outputs of early, prompt and late ones are all used in this discriminator which results in the lowest baseband computational load and produces nearly true error output within $1 / 2$ chip of input error for $1 / 2$ chip correlator spacing[3]. So here sets 0.5-chip spacing between the early, prompt, and late correlators. As the carrier and code is coherent, code tracking loop can use the output of the carrier tracking loop to aid the code tracking loop. In the structure, carrier aiding part is the point studied in this paper. In the way, because of the aid of carrier tracking loop, the code loop is able to keep stable even at the high dynamic condition.

\subsection{Signal}

The received frequency signal $\mathrm{r}(\mathrm{t})$ and the local signal ${ }^{U_{o}(t)}$ generated by the carrier tracking loop could be described respectively as

$$
\begin{aligned}
& r(t)=A \cos \left(2 \pi\left(f_{t}(k)+f_{d}(k)\right) t+\theta\right) D(t-\tau) C\left(R_{c}(t)+R_{d}(t)-\tau\right)+n(t) \\
& U_{o}(t)=\exp \left(\left(2 \pi\left(f_{i n}(k)+f_{d}(k)\right) t+\theta\right)\right.
\end{aligned}
$$

Where

A is the signal amplitude, 
$k$ is the sequence number of carrier frequency.

$f_{t}(k), f_{d}(k)$ and $\theta$ denote respectively the kth carrier frequency transmitted according to the frequency hopping pattern, Doppler frequency offset during the kth hop dwell time which is related to the frequency hopping pattern and the original phase of the carrier. $f_{d}(k), \theta$ are the estimates of $f_{d}(k)$ and $\theta$.

$D(t)$ is the non-return-to-zero (NRZ) data signal, $\mathrm{D}(\mathrm{t})=1$ is set in the code tracking procedure in this paper,

$C(t)$ is the received PN code,

$R_{c}(t), R_{d}(t)$ denote respectively the code rate and the code Doppler caused by the higher dynamics,

$\tau$ is the transmission delay from the satellite to the receiver.

$n(t)$ is the received noise that assumed to be Gaussian and independent of the useful signal.

$U_{o}(t)$ is the output of the numerical controlled oscillator of the carrier tracking loop.

The early, prompt, and late outputs of the correlators respectively are described as:

$$
U_{i}=a \exp \left(j\left(f_{e} t+\theta_{e}\right)\right) R_{i}\left(\tau_{i}\right)+\mu_{i}, i=E, P, L
$$

Where

$$
\begin{gathered}
R_{i}\left(\tau_{i}\right)=\frac{1}{T} \int_{(n-1) T}^{n T} C_{i}\left(t-\tau_{i}\right) C_{r}(t) d t \\
=I_{E}+Q_{E} \\
\mu_{i}=\int_{(n-1) T}^{n T} n(t) \exp \left(\left(2 \pi\left(f_{t}(k)+f_{d}(k)\right) t\right) C_{r}(t) d t\right. \\
=n_{I}+j n_{Q} \\
\tau_{i}= \begin{cases}\hat{\tau}-\frac{D}{2}, & i=L \\
\hat{\tau}, & i=P \\
\hat{\tau}+\frac{D}{2}, & i=E\end{cases}
\end{gathered}
$$

I, Q mean in-phase component and quadra-phase component respectively. E, P, L denote early, prompt, late. Symbol ${ }^{f_{e}}$ means the estimate of $f_{e}$. $\theta_{e}$ denotes corresponding estimated errors and ${ }^{\mu_{i}}$ is complex noise signal; D is the normalized discriminator spacing scaled by code width, and $\mathrm{T}$ is the integration time. As ${ }^{\mu_{i}}$ is independent of the signal, the following deduction excludes $\mu_{i}$.

The formula of the discriminator [6] is

$$
\delta=\frac{1}{4}\left(\frac{I_{E}-I_{L}}{I_{P}}+\frac{Q_{E}-Q_{L}}{Q_{P}}\right)
$$

So the output of the discriminator is 


$$
\begin{aligned}
e_{c} & =\frac{R\left(\tau_{e}+\frac{1}{2} D\right)-R\left(\tau_{e}-\frac{1}{2} D\right)}{R\left(\tau_{e}\right)} \\
& \approx \frac{\left(1-\frac{\left|\tau_{e}+\frac{1}{2} D\right|}{D}\right)-\left(1-\frac{\left|\tau_{e}-\frac{1}{2} D\right|}{D}\right)}{1-\frac{\tau_{e}}{D}} \\
& =\frac{2 \tau_{e}}{1-\left|\tau_{e}\right|} \approx 2 \tau_{e}
\end{aligned}
$$

The structure of the second-order loop filter [3] is shown in Fig. 2. The expression of the filter is provided in (5).

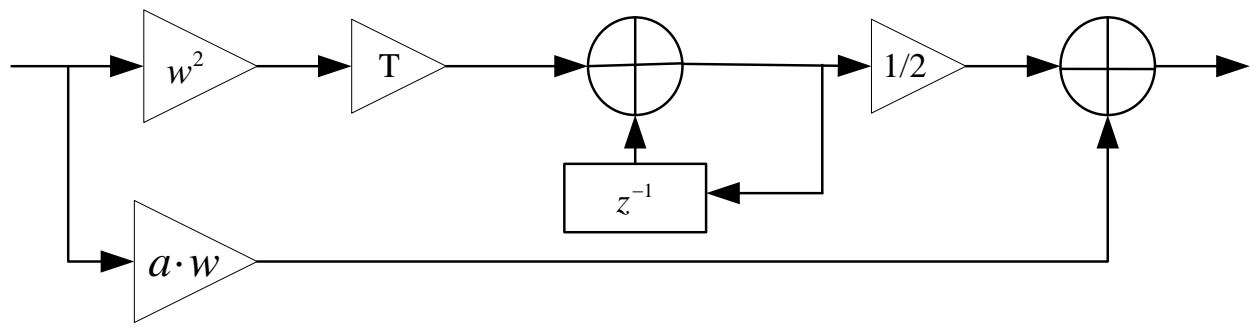

Fig. 2. second-oder loop filter

$$
F(z)=a w+\frac{w^{2} T}{2\left(1-z^{-1}\right)}
$$

Where

$w$ is the natural radian frequency of the code tracking loop;

$T$ is the integration time;

After the loop filter, an accumulator which accumulates the code frequency word, exists. The result of the accumulator is used to check out the code table to generate the prompt, early and late code.

\section{The Scheme of Code Tracking Loop Aided by Frequency Hopping Pattern}

Here suppose the scenario in which, after code and carrier acquisition procedure [7], and the carrier tracking loop, the carrier of the signal can be taken off precisely. Then the main work is to track the PR code accurately. The carrier loop output should always provide aiding to the code loop because the carrier loop jitter is much less noisy than the code loop jitter and thus more accurate. The carrier loop aiding removes virtually almost all of the line-of-sight dynamics from the code loop, so the code loop filter order can be made smaller and the predetection integration time can be made longer, and the code loop bandwidth can be made much narrower than 
for the unaided case, thereby increasing the code loop tracking threshold and reducing the noise in the code loop measurements [3]. Therefore the main work of this paper is to eliminate the impact of $R_{d}(t)$ with the help of the carrier tracking loop according to the frequency hopping pattern which is known beforehand.

The relation between code rate, carrier frequency, carrier Doppler frequency and code Doppler, is shown as

$$
\frac{R_{d}(t)}{f_{d}(k)}=\frac{R_{c}(t)}{f_{t}(k)}
$$

$f_{t}(k)$ and $f_{d}(k)$ are both changed according to the carrier frequency hopping pattern. Though $f_{d}(k)$ is also decided by the dynamics of the receiver, ${ }^{f}(k)$ can be gotten from the carrier tracking loop before code tracking loop starts work. $R_{d}(t)$ is only influenced by the receiver's line-of-sight dynamics and bears no relation of the carrier frequency hopping pattern. Code rate $R_{c}(t)$ is a constant decided by the signal emitted by the satellite.

If there is no carrier frequency hopping such as the Direct Sequence Spread Spectrum System, ${ }^{R_{c}(t)}$ and $f_{d}(t)$ have an fixed relationship as

$$
\lambda=\frac{R_{c}(t)}{f_{d}(t)}
$$

Where $\lambda$ is a constant, because of the fixed relation between the code rate and the fixed carrier frequency, which are coherent in the transmitter.

However as the carrier frequency $f_{t}(k)$ emitted is changed according to some hopping pattern given by the designer in Direct Sequence/Frequency Hopping (DS/FH) hybrid spread spectrum system, (7) should be changed to (8).

$$
\lambda(k)=\frac{R_{c}(t)}{f_{d}(k)}
$$

Here a parameter $\lambda(k)$ which is changed according to the frequency hopping pattern can be used to make a product with $f_{d}(k)$ to get $R_{d}(t)$.

$$
R_{d}(t)=\lambda(k) f_{d}(k)
$$

$R_{d}(t)$ and $f_{d}(k)$ are the estimates of $R_{d}(t)$ and $f_{d}(k)$ respectively. When $R_{d}(t)$ is gotten, it can be added into the code tracking loop shown in Fig. 1. In this way, the impact of the dynamics in the code tracking loop can be decreased. So we can use narrow loop bandwidth and less-order loop filter to get required code tracking precision. 


\section{Simulation and Analysis}

Based on the analysis above, firstly simulation of the impact of the carrier frequency hopping on the code tracking loop is provided. Secondly, code tacking loop aided by the frequency hopping pattern is simulated and analyzed under different carrier to noise ratios in this section. Noise is assumed to be Gaussian White noise and independent of the useful signal.

Parameters used in the simulation's are described in the following: code rate is $1.023^{M c p s \text { (mega-chips-per-second) }}$, the number of chips in a code period is 1023 , the frequency hopping period is $1 \mathrm{~ms}$, the radio frequency range is $2.2 \sim 2.7 \mathrm{GHz}$, the code tracking loop bandwidth is $1 \mathrm{~Hz}$, the carrier tracking loop bandwidth is $10 \mathrm{~Hz}$, the integral time is $1 \mathrm{~ms}$, and velocity is $6 \mathrm{~km} / \mathrm{s}$.

Fig. 3 shows the carrier frequency hopping pattern emitted by the transmitter in one hopping period. The duration of one hopping cycle is $10 \mathrm{~ms}$.

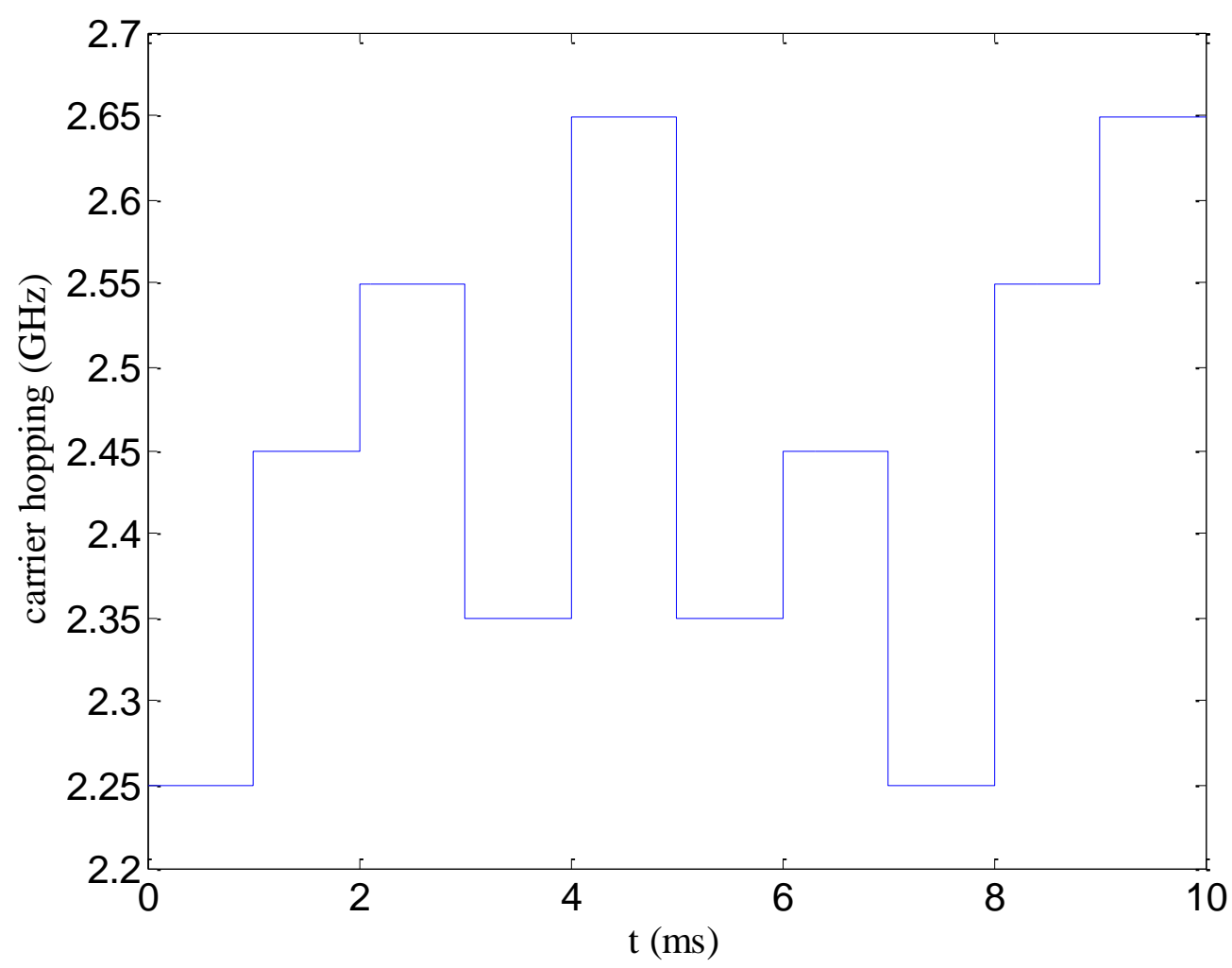

Fig. 3. Carrier hopping pattern

The result of the code tracking loop under no noise, with the residual frequency which is called the Doppler frequency agility caused by the carrier hopping is shown in Fig. 4. 


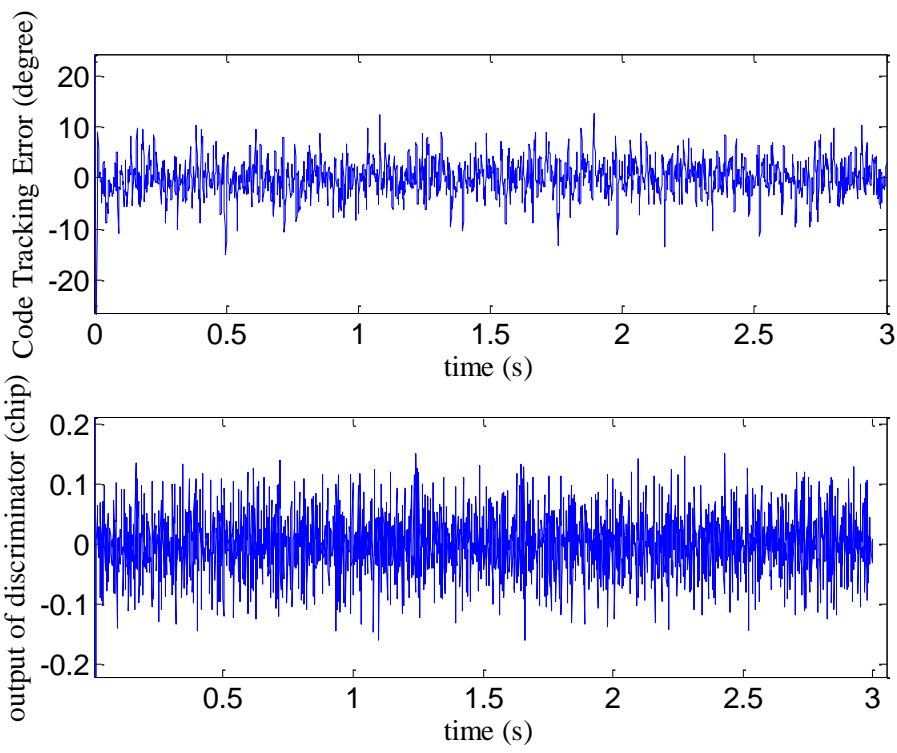

Fig. 4. Errors with Doppler frequency agility

From Fig. 4, it is obvious to see that the errors of the tracking loop are big when the frequency agility existed. The Doppler frequency agility can be removed by the method described in [1]. Then the code tracking loop can work with much lower residual frequency.

Fig. 5 shows the code tracking result without noise using this carrier aid method. The tracking error is very small and the precision is enough for the application in Telemetry, Tracking and Command (TT\&C) system.

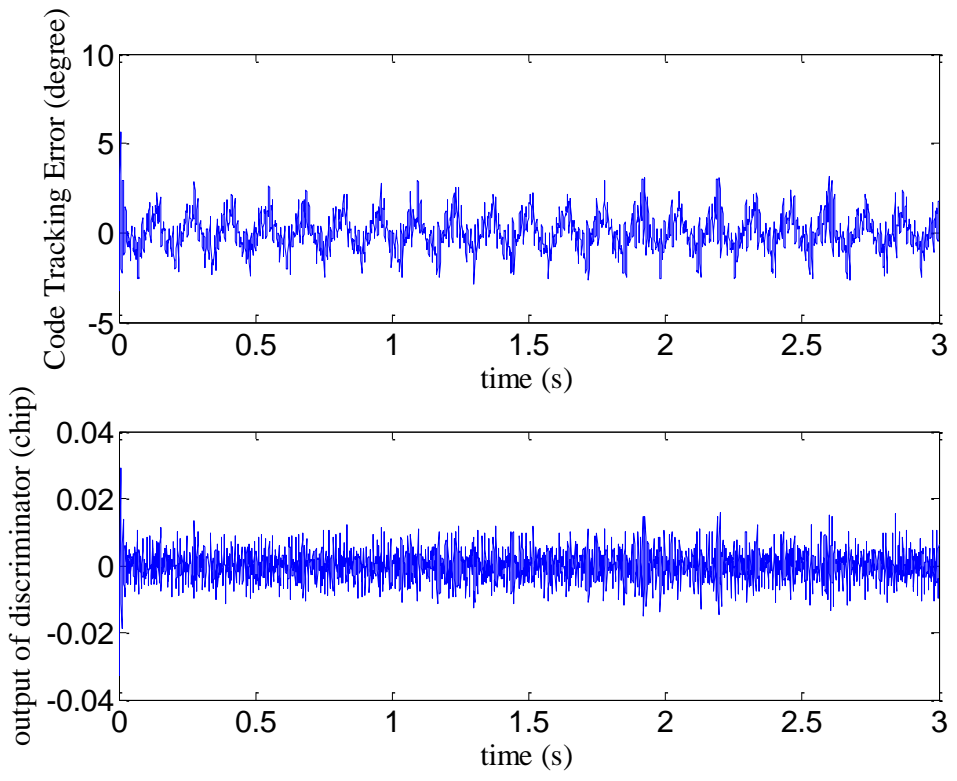

Fig. 5. Tracking result without noise 
The code tracking 1-Sigma jitter under different carrier to noise ratios with different code tracking loop bandwidths is provided in Fig. 6 .

From Fig. 6, we can see with the decrease of the bandwidth, 1-Sigma jitter of code tracking loop drops markedly. When the code loop bandwidth is $0.1 \mathrm{~Hz}$, the 1-Sigma jitter is small enough for the application. The method described in this paper allowed code tracking loop uses narrow bandwidth. In the situation with the same bandwidth, if the carrier to noise ratio of the received signal increases 1-Sigma jitter decreases.

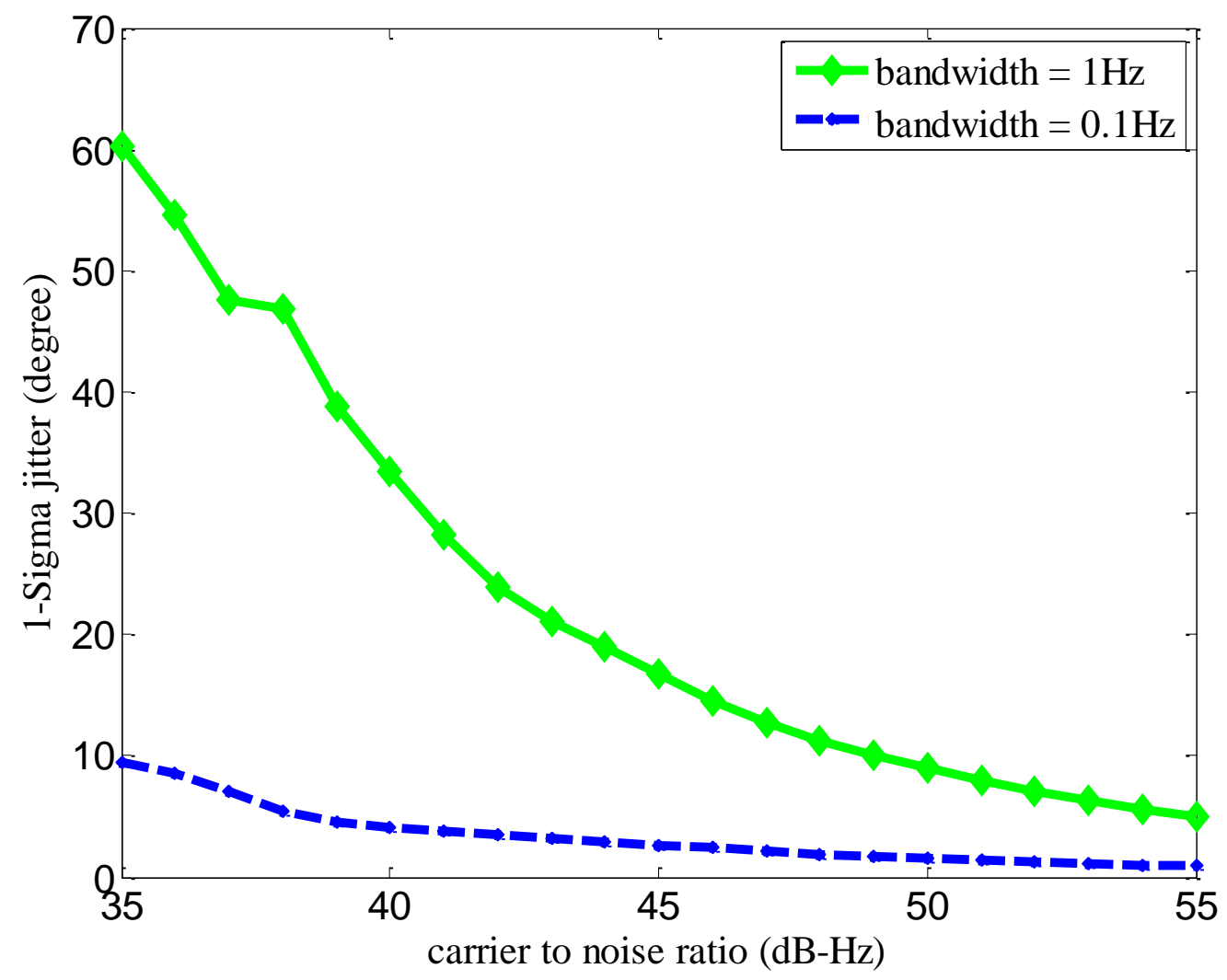

Fig. 6. Delay lock loop jitter versus $\mathrm{C} / \mathrm{N}$

\section{Conclusion}

In this paper, we present one scheme on the code tracking loop aided by carrier tracking loop with the Direct Sequence/Frequency Hopping Spread Spectrum (DS/FH) hybrid spread spectrum signal in the Telemetry, Tracking and Command application. This method can effectively decrease the impact of the dynamics on code tracking loop, so narrow bandwidth and less-order filter can be used. Based on this method, the simulations under different carrier to noise ratios show that the method is valid. From the simulations we can know that when the bandwidth of the code tracking loop is set smaller, the results of the code tracking loop are better. The future work following this paper should be investigating the best code tracking loop bandwidth. 


\section{References}

[1] CHEN Zhu, WANG Yuan-qin and YANG Wen-ge and WANG Zai-yuan "Research on Carrier Tracking in Hybrid DS/FH Spread Spectrum TT\&C System", 2010 2nd International Conference on Future Computer and Communication, IEEE, pp. 796-800.

[2] Shengyun Meng, Wenge Yang, Weitao Lu, Jia Liu, Jinfeng Yu "Code Tracking Performance of DS/FH Spread Spectrum Signal for TT\&C", 2010 2nd International Conference on Information Management and Engineering, v2, IEEE, pp. 491-495.

[3] Elliott D.Kaplan,Understanding GPS Principles and Applications, Second Edition. Artech House, 2006.

[4] A. J. Van Dierendonck, P. Fenton and T. Ford, "Theory and Performance of Narrow Correlator Spacing in a GPS Receiver", Journal of the Institute of Navigation, Fall 1992, 265-283.

[5] M. K. Simon, "Noncoherent Pseudonoise Code Tracking Performance of Spread Spectrum Receivers", IEEE TRANSACTIONS ON COMMUNCATIONS, 1997, 327-345.

[6] Gang Xie, Principles of GPS and Receiver Design. Publishing House of Electronics Industry, 2009 (in Chinese).

[7] Yang Wen-ge, Meng Sheng-yun, Wang Jin-bao, et. al, "Acquisition Performance Analysis of a Synchronization Scheme of DS/FH Hybrid Spread Spectrum Signals for TT\&C," Proc. ICEMI, Vol. IV, 2009, pp. 395-399. 